\title{
CARDIOGRAPHIC PATTERNS IN SYSTOLIC AND DIASTOLIC OVERLOAD OF THE LEFT VENTRICLE
}

\author{
BY \\ LUDWIK SEDZIWY AND JOHN SHILLINGFORD
}

From the Postgraduate Medical School of London

Received January 23, 1961

In 1952 and subsequently $(1959,1960)$, Cabrera and his associates advanced the theory that the effect of diastolic overload of the left ventricle, such as occurs in mitral or aortic regurgitation, would have a different effect on the cardiogram from that produced by systolic overload, as in hypertension and aortic stenosis. They stated that in cases of diastolic overload there was an increase of the vectorcardiographic $Q$ loop to the right and anteriorly, and increase in the $R$ loop backwards and to the left, and that the J-point was displaced forward and to the right, while the $\mathrm{T}$ loop kept a normal orientation with the QRS loop. On the other hand, in cases of systolic overload the Q loop became small or disappeared. The $R$ loop increased and became displaced posteriorly, the $S$ loop disappeared or became straightened, the J-point displacement was greater and the T loop moved forwards and towards the right. These changes appear in the electrocardiogram as follows: in diastolic overload there is a $Q$ wave, a tall $R$ wave and an upright, peaked symmetrical $T$ wave in left ventricular leads; and the $Q$ wave of left ventricular leads is reflected in a primary $R$ wave in the right-sided chest leads : in systolic overload the $\mathbf{Q}$ wave is absent or small, there is a high $\mathbf{R}$ wave, $\mathrm{S}-\mathrm{T}$ depression, and $\mathrm{T}$ wave flattened or inverted.

We have selected a group of patients in whom on clinical grounds we considered that there was either pure diastolic overload or pure systolic overload of the left ventricle, and compared the cardiographic patterns in these two conditions.

\section{METHODS}

A drawing apparatus of the type first described by Shillingford and Brigden (1951) was used for the construction of the vectorcardiogram from the scalar electrocardiogram. The electrode position of the cube system of Grishman et al. (1949) was used. The electrocardiographic records were taken with an Elmqvist three-channel machine at a paper speed of 100 millimeters per second. The mechanical scanning was made at time intervals of $1 / 200$ of a second for the QRS and $1 / 50$ of a second for the $T$ wave. The frontal, horizontal, and sagittal loops were drawn as though viewed from the front, top, and left side of the patient.

Eleven patients were studied, five of whom were judged on clinical and hæmodynamic grounds to have an increase in left ventricular output without a greatly raised pressure, and six in whom there was a high left ventricular pressure without an increase in output. The former included three patients with mitral regurgitation, one with aortic regurgitation, and one with mixed mitral and aortic regurgitation. The latter included four with severe hypertension, one with acquired aortic valvular stenosis, and one with severe coarctation of the aorta.

\section{RESULTS}

Stroke Volume Overload. Fig. 1 shows the vectorcardiogram and electrocardiogram of a patient with diastolic overload. 
A woman of 56 had rheumatic fever at age of ten. She had chronic heart trouble 14 years ago and at that time was complaining of dyspnœa. Two years ago chest pain and occasional palpitation were experienced on exertion. On examination, the pulse was regular at 75 a minute and the blood pressure was 150/90. The jugular venous pressure not raised. The apex beat was in the fifth intercostal space in the anterior axillary line. There was a loud pansystolic murmur at the apex conducted to the axilla, a third heart sound, and a short mid-diastolic murmur. An aortic early diastolic murmur was also present. X-ray of the chest showed moderate enlargement of the left atrium and left ventricle. A diagnosis of mitral regurgitation and aortic regurgitation was made.

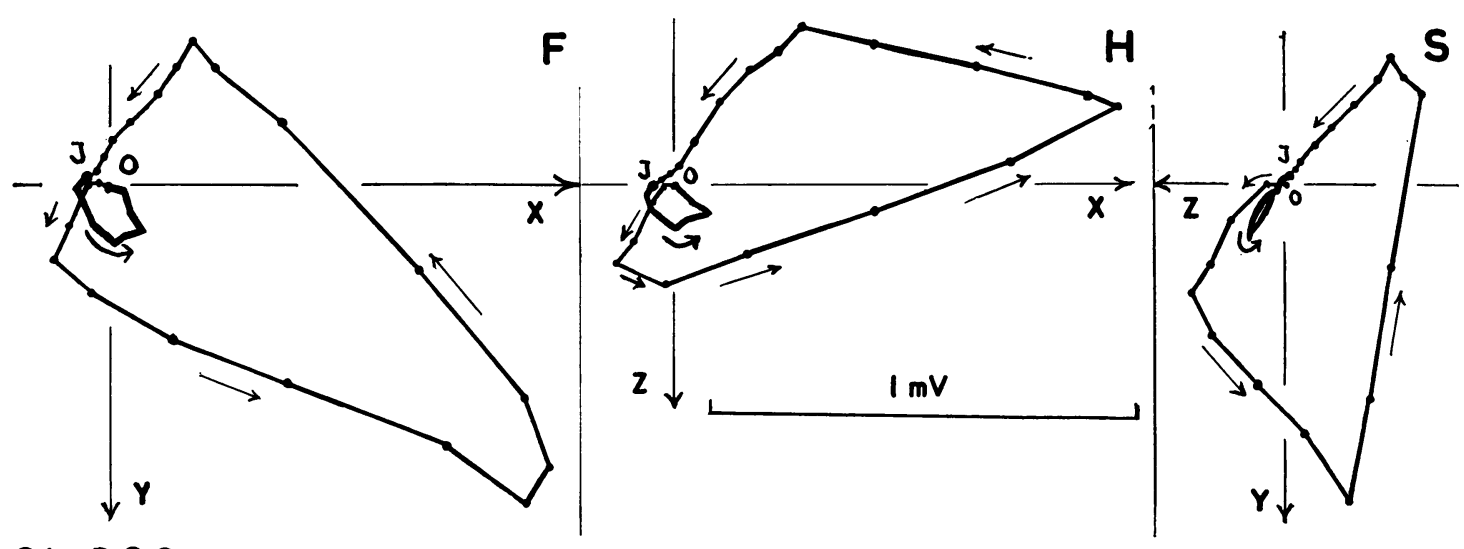

214322.

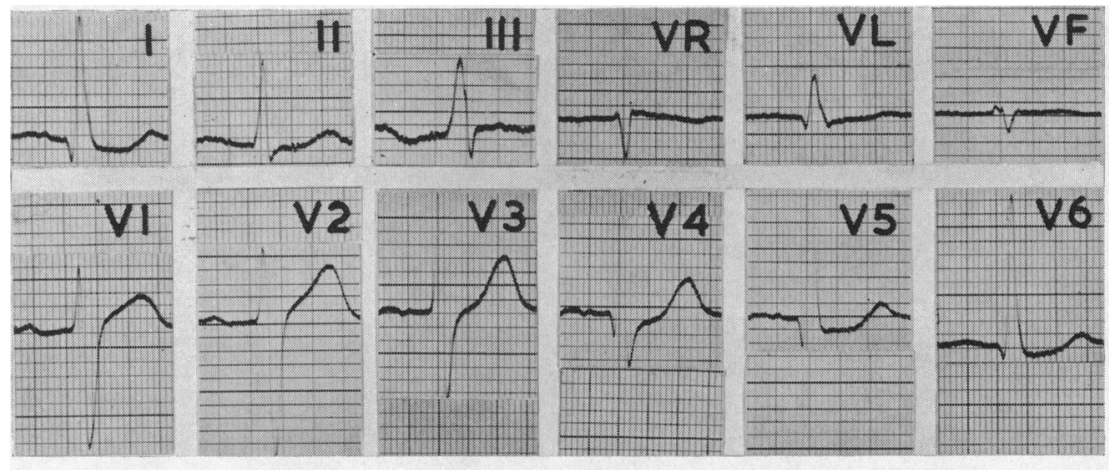

Fig. 1.-The vectorcardiogram and electrocardiogram from a patient with chronic rheumatic heart disease, mitral and aortic regurgitation being the predominant disorders.

The QRS loop in the ventorcardiogram showed counter-clockwise rotation on the frontal, horizontal and sagittal planes with no signs of slurring or notching (Fig. 1). The Q loop was moderately enlarged. The J-point was not much displaced and the angle between the mean axis of QRS loop and T loop was normal on three planes. The electrocardiogram showed a small $\mathrm{Q}$ wave in leads I, VL, V4, V5, V6, corresponding with the initial $R$ wave in V1. The $R$ waves in leads I, VL, V5, V6 were prominent. The RS-T segment showed slight depression, but the $\mathrm{T}$ waves were upright in the bipolar limb leads and in all chest leads (V1 to V6) and flat in VR, VL, VF. This picture corresponds with the typical pattern of diastolic overload of the left ventricle as described by Cabrera and Munroy (1952).

Three other patients with diastolic overload showed patterns similar to those of Fig. 1 except for a figure of eight shape of QRS loop on the frontal plane with counter-clockwise rotation of the main 
portion in one and slight deviation of $\mathrm{T}$ loop in a second case. References to these cases will be made later.

In Fig. 2 are shown the vectorcardiogram and electrocardiogram from a patient with severe hypertension in whom the cardiographic patterns resembled those found in diastolic overload.
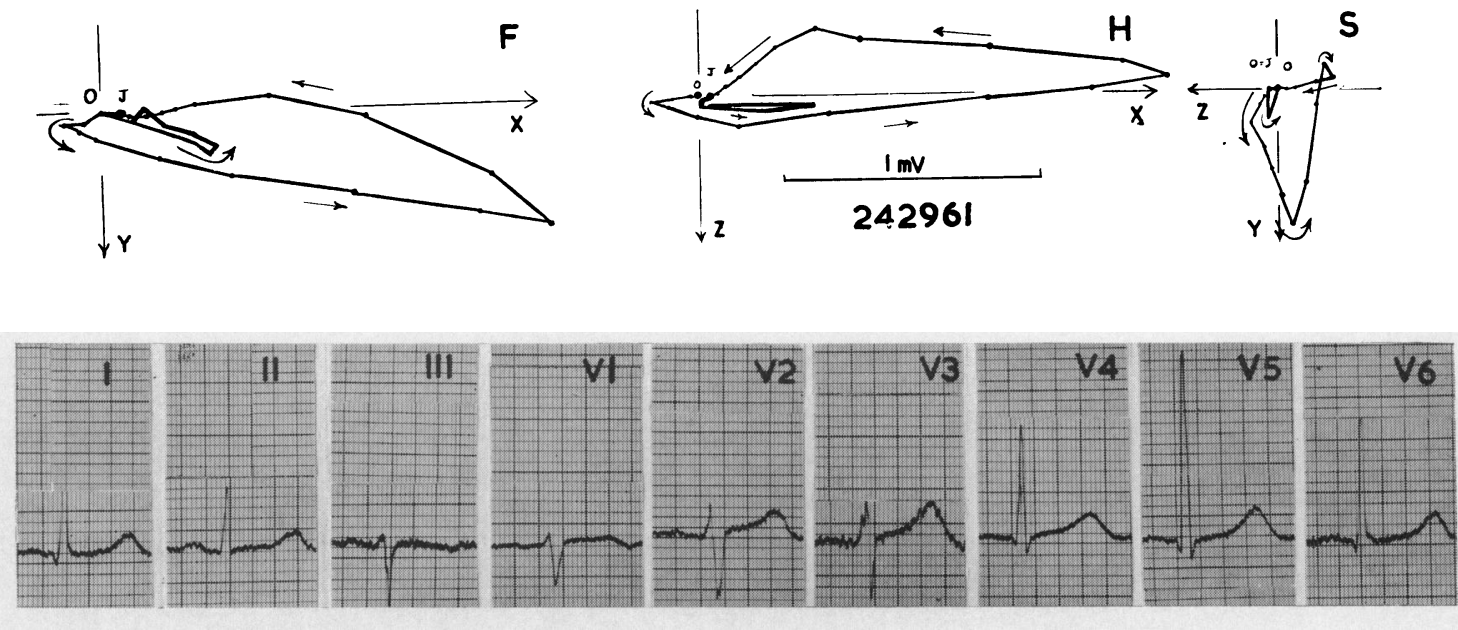

FIG. 2.-The vectorcardiogram and electrocardiogram from a patient with hypertension.

This man of 59 had been discharged from the army because of hypertension 14 years before he came under observation. He was treated in 1959 with bretylium tosylate and thereafter required increasing doses. For six years he had had severe headaches and nocturia, and for one year mild dyspnœa on exertion. On examination the pulse was 72 and regular and the blood pressure was 260/175. The apex beat was heaving and situated in the anterior axillary line. A variable atrial sound was heard at the apex and there was a grade 1 ejection systolic murmur. The X-ray of the chest showed gross left ventricular enlargement and slight left atrial enlargement. The vectorcardiogram showed a well developed $Q$ loop and a normal angle between the mean axes of the QRS and T loops-smaller than $90^{\circ}$ on three planes. The electrocardiogram revealed left axis deviation of QRS, a small Q wave in leads I, V5, and V6, with upright T waves.

Systolic Overload. The vectorcardiogram and electrocardiogram of a patient with left ventricular systolic overload are shown in Fig. 3.

A man of 31 was found one year ago to have high blood pressure. He was admitted to Hammersmith Hospital for investigation. On examination there was increased arterial pulsation in the neck and arterial pulses were felt over the back involving mainly the subscapular and intercostal vessels. A soft delayed systolic murmur was heard in the fourth intercostal space at the left sternal edge. A third sound was present at the apex. The aortic second sound was loud. The blood pressure in the right arm was 230/130, and in the left arm 210/115. The femoral pulse palpable but diminished. The sytolic blood pressure in the legs was 150 on both sides. An X-ray of the chest showed no significant increase in the cardiac size, a prominent ascending aorta, an abnormal aortic knucle, and rib notching on both sides. The diagnosis of severe coarctation of the aorta was confirmed at operation.

The QRS loop on the vectorcardiogram showed counter-clockwise rotation on the frontal, horizontal and sagittal planes without any slurring or notching. The Q-loop was present but not augmented. The Jpoint was not displaced. There was marked deviation of the $\mathrm{T}$ loop anteriorly and toward the right with an increase of the angle between the mean axes of the QRS and T loops. The electrocardiogram showed diminution of the $Q$ wave and negative $T$ wave in leads $I, V L, V 5$, and V6. This picture corresponded with the typical pattern of systolic overload of the ventricle described by Cabrera.

A pattern similar to that in Fig. 2 was found in three other cases of diastolic overload with the exception that clockwise rotation on the frontal plane was present in one instance. The vectorcardio- 

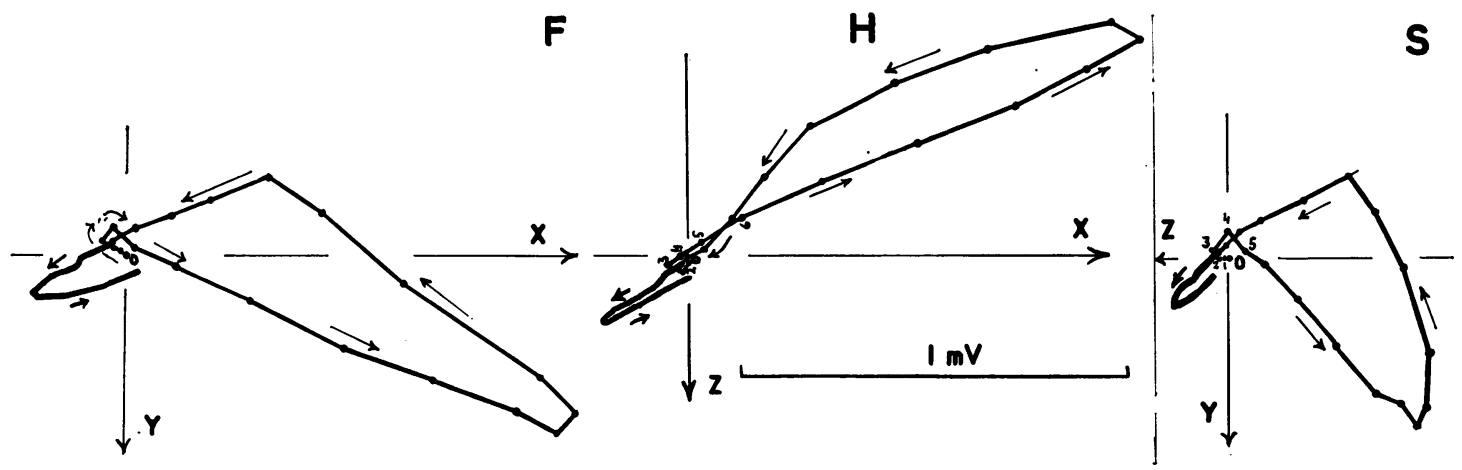

246569

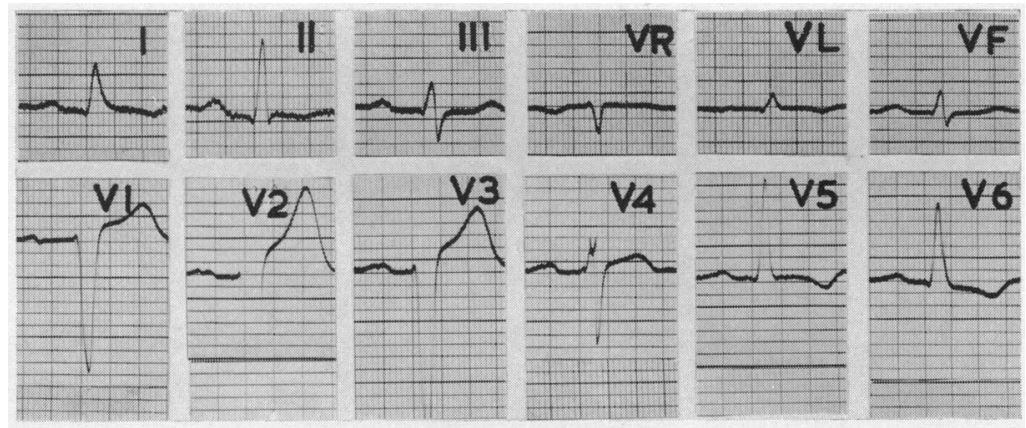

FIG. 3.-The vectorcardiogram and electrocardiogram from a patient with hypertensive heart disease.
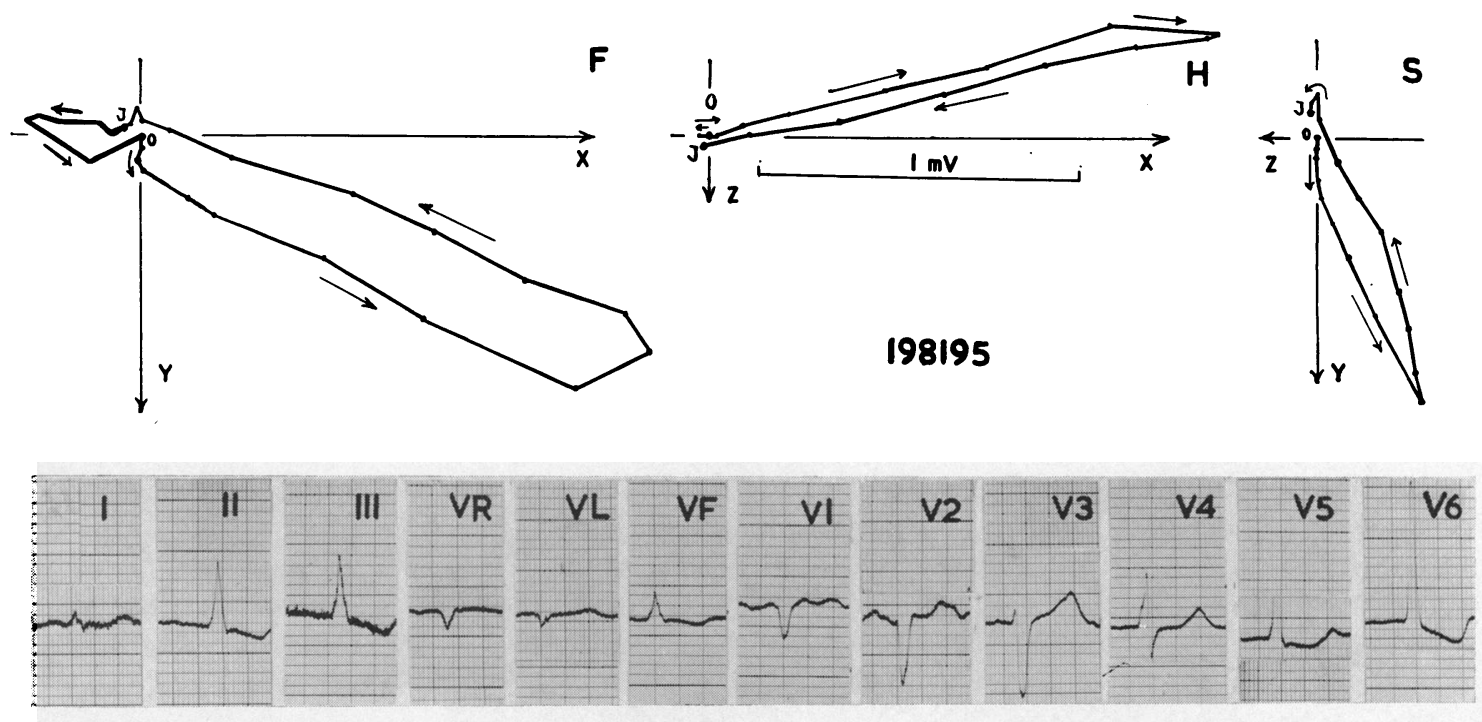

Fig. 4.-The vectorcardiogram and electrocardiogram from a patient with chronic rheumatic heart disease and predominant mitral regurgitation. 
gram and electrocardiogram from another patient with severe mitral regurgitation in whom the patterns resembled those found in systolic overload are shown in Fig. 4.

A woman aged 31 had acute rheumatic fever at age of eleven. Three years before she came under observation she had been seen at Hammersmith Hospital with mitral valve disease and regurgitation. Two weeks before admission she became excessively tired, and noticed ankle swelling and reduction in exercise tolerance. On examination, atrial fibrillation was present with a heart rate of 90 a minute. The blood pressure was $130 / 70$, the jugular pressure was raised $2 \mathrm{~cm}$. above the clavicular level. The apex beat was in the sixth space outside the mid-clavicular line. There was a heaving left ventricle. The first sound was loud with a Grade 3 pansystolic murmur radiating out into the axilla; a third heart sound and a short mid-diastolic murmur were also heard at the apex. The X-ray of the heart showed enlargement of the left ventricle and great enlargement of the left atrium. The diagnosis of rheumatic heart disease with severe mitral regurgitation was made.

The vectorcardiogram showed the absence of $\mathrm{Q}$ loop, clockwise rotation on the horizontal, and marked displacement of the T loop in the direction opposite to that of the QRS loop. In the electrocardiogram these changes corresponded to the absence of Q deflection and negative T waves in leads II, III, VF, and V6.

The cardiograms of four cases judged on clinical and hæmodynamic grounds to be examples of diastolic overload are shown in Fig. 5, also shown in this figure are the records of four patients suffering with pure systolic overload of the left ventricle. The comparison between the electrocardiographic patterns reveals that the patterns of diastolic overload are present not only in cases with clinical diastolic overload (A, B, C) but also in systolic overload (E). On the contrary,

DIASTOLIC OVERLOAD

A.

II

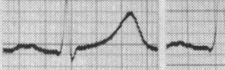

III
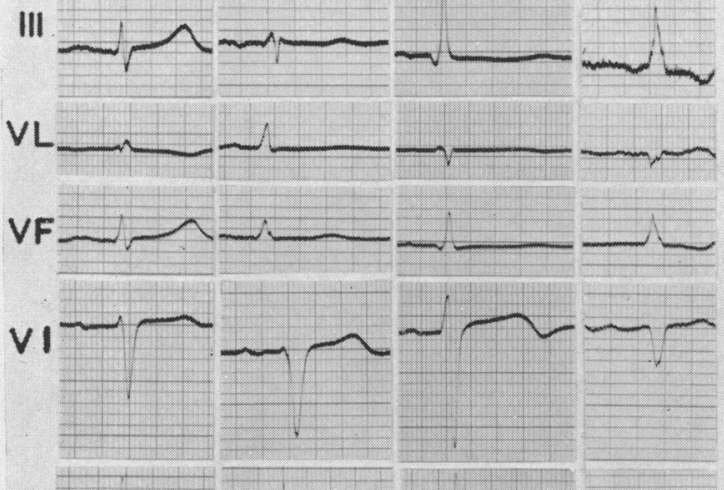

V5

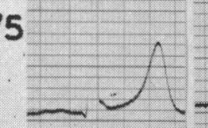

V6

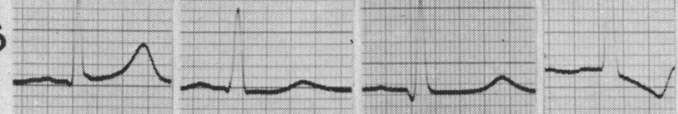

\section{SYSTOLIC OVERLOAD}

E. F.

G.

$\mathrm{H}$.
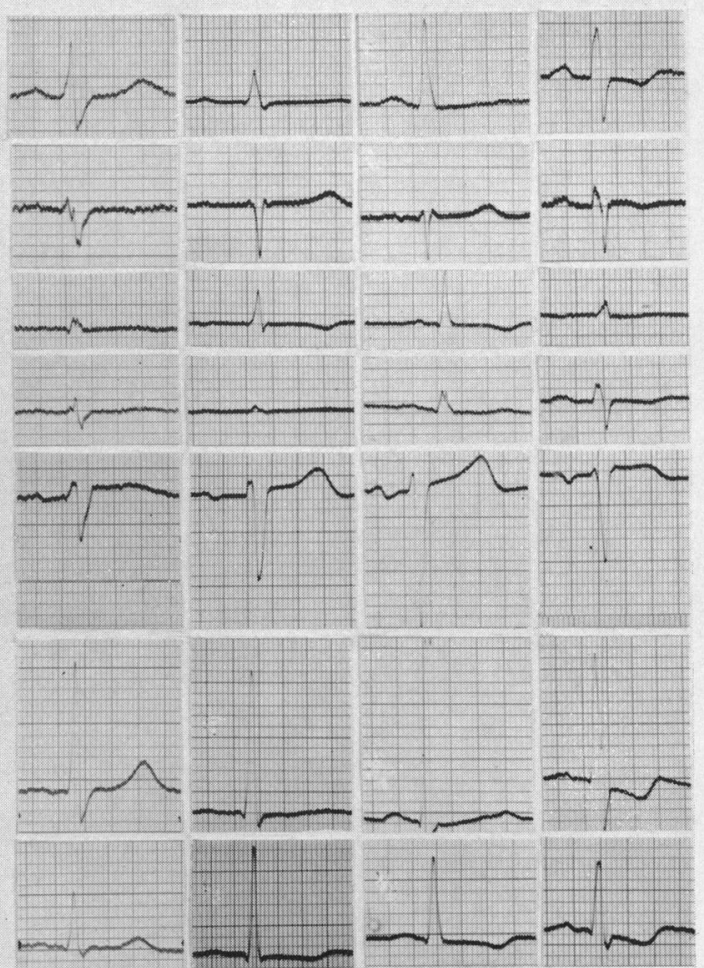

FIG. 5.-Electrocardiograms from patients with diastolic overload (A, B, C, D) and systolic overload (E, F, G, H), showing overlapping of groups. 
diminution or absence of a $Q$ wave and negative $T$ waves in left ventricular leads regarded as characteristic of systolic overload were found in diastolic overload (D).

\section{Discussion}

Although the theory of Cabrera that the cardiographic patterns of diastolic and systolic overload should be different is attractive, and the results of Deglaude and Laurens (1958) tend to confirm this idea, Selzer et al. (1958) were not able to find any practical differences between the two in the electrocardiogram.

Our own results show that there may be considerable overlap between the two groups. We have, for example, found patients with severe hypertension in whom the cardiographic pattern would fulfil the criteria claimed by Cabrera as denoting diastolic overload. On the other hand, we have found also inversion of $T$ waves and absence of $Q$ waves in the left ventricular leads in patients with severe valvular regurgitation. Cabrera has stated that in severe cases of diastolic overload there may be $\mathrm{T}$ wave inversion. In practice, other factors must play a part. Foremost amongst these is the state of the coronary arteries and the resultant coronary arterial flow. Thus, in aortic regurgitation and stenosis the blood flow through the coronary arteries deviates from the normal. Moreover, the administration of drugs and disturbance in the electrolyte balance may influence the clinical and cardiographic picture.

We have not made a statistical survey with a larger number of patients, and it could well be that on average there are essential differences in the cardiogram between the two conditions causing left ventricular hypertrophy. However, in our small series we have found enough evidence to cause us to have doubts about its diagnostic significance.

\section{SUMMARY}

The vectorcardiogram and electrocardiogram of patients with left ventricular hypertrophy have been studied. These have been divided into two groups; the first includes those with an increased left ventricular stroke output without a raised pressure (diastolic overload), and the second includes those with increased left ventricular pressure with a normal stroke output (systolic overload). There appears to be considerable overlap in the cardiographic patterns in the two groups, and it has not been possible to separate them clearly in clinical practice. The reasons for this are discussed.

We should like to thank the Consultant Physicians of Hammersmith Hospital for allowing us access to the patients and Miss Powell for her technical assistance.

\section{REFERENCES}

Cabrera, E., and Monroy, J. R. (1952). Amer. Heart J., 43, 661. (1952). Amer. Heart J., 43, 669.

and Gaxiola, A. (1959). Prog. Cardiovasc. Dis., 2, 219.

- , and Gaxiola, A. (1959). Prog. Cardio

Deglaude, L., and Laurens, P. (1959). Arch. Mal. Caur, 52, 263.

Grishman, A., and Scherlis, L. (1952). Spatial Vectorcardiography. W. B. Sauders Co.

Selzer, A. (1958). Amer. J. Med., 24, 831.

, Naruse, D. Y., York, E., and Kahn, K. A. (1958). Circulation, 18. 780.

Shillingford, J., and Brigden, W. (1951). Brit. Heart J., 13. 233. 